formation de peaux superficielles à l'abri desquelles l'hydrolyse se développe ;

$7^{\circ}$ Obtention d'une caséine de qualité supérieure.

\title{
RÉFÉRENCES
}

[1] W. H. SHEFFieLd, Brevet a mériea in 1.716 .799 du 11 juin 1929

[2] R. W. Змгтн. Ind. Eng. Chem., t. XXVI, 1934, p. 819.

[3] Maurice BEAu. La caséine, $1^{\text {re }}$ et $2^{\circ}$ partie. Le Lait, éditeur, 1932.

[4] F. L. ChappeLL. Brevet a méricain 1.892.233 du 27 déce mbre 1932.

\section{LES SAVEURS ET ODEURS ANORMALES DU LAIT}

$$
\text { par }
$$

Jean PIEN

Docteur ès-Sciences

Ingénieur chimiste (I.C.R.)

Directeur des Laboratoires des " Fermiers Réunis"

\author{
et \\ S. HERSCHDOEREER \\ Docteur ès-Sciences
}

(Vienne)

(Fin)

\section{L'HYDROLYSE DIASTASIQUE DE LA MATIËRE GRASSE DU LAIT}

Nous sommes toujours ici dans ce que nous avons appelé le cinquième groupe de causes des saveurs et odeurs anormales du lait, consistant en des modifications d'origine diastasique des éléments normalement contenus dans le lait. Nous avions divisé ces altérations en deux groupes : les oxydations qui mènent au goût d'huilage (que nous avons étudiées) et l'hydrolyse qui mène à la rancidité dont nous allons maintenant dire quelques mots.

Il est nettement établi depuis les travaux classiques de Porcher que la rancidité est bien un phénomène d'hydrolyse. Nous savons d'autre part que l'hydrolyse de la matière grasse du lait consiste en une décomposition des graisses en glycérine et acides gras parmi lesquels l'acide butyrique, cause du goût de rance et même d'une certaine saveur amère.

Nous savons encore que cette hydrolyse, qui peut être l'œuvre de certaines bactéries, est le plus souvent d'origine diastasique et nous connaissons la diastase responsable : la lipase.

a) Présence de la lipase dans le lait. - Le lait de vache normal paraît être assez pauvre en lipase. Il n'y a pas concordance de vues chez les divers auteurs à ce sujet. Pour Leroy et S. PaLmer [48] la lipase serait absente dans le lait de vache normal et n'existerait que dans les laits de lactation avancée à la suite d'une lipogénèse plus ou moins intense au niveau de la glande mammaire. DAviEs au contraire considère comme normale, dans le lait de vache, l'existence d'une lipase spécifique. 
DORNER et WIDMER [49] croient également à l'existence d'une ou même de deux lipases distinetes dans le lait de vache normal et ils estiment que la quantité de lipase varie d'une vache à l'autre.

Tout le monde est d'aecord en tout eas pour affirmer que le lait de vache peut contenir, en plus ou moins grande abondance, une lipase responsable de la libération d'acides gras dans le lait ou ses dérivés.

b) Mode d'action de la lipase. - Nous n'entreprendrons pas de faire ici la théorie de l'action de cette diastase. Bornons-nous à citer quelques-unes des observations les plus intéressantes rencontrées dans la littérature des laits de mauvais goût.

Proks [50] lie la question de l'hydrolyse lipasique à la plus ou moins grande teneur du lait en acide oléique. Il estime que, fréquemment, l'hydrolyse peut résulter de la coopération des microbes du groupe Bact. fluorescens liquefaciens.

Le curieux travail de Davies nous apprend que, contrairement à ce qui se passe pour les oxydases, des traces de métaux lourds inhiberaient l'action de la lipase. Mais l'étain et l'aluminium, qui ne catalysent pas les oxydations diastasiques, ne s'opposeraient pas à l'action de la lipase. On en déduirait done que la destruction de la. lipase par les métaux est proportionnelle à la faculté que possèdent ces métaux d'activer l'oxygène. De là à eonclure que la lipase est hautement oxydable et même peut être anti-oxydante, il n'y avait qu'un pas que Davies a franchi. Ce qui nous intéresse le plus ici serait de tirer des travaux de DAviES, s'ils sont confirmés, la conclusion assez inattendue que la rancidité et l'huilage-suiffage sont deux défauts qui peuvent être incompatibles dans certains cas. Nous nous garderons bien d'aller plus loin dans cette voie. De nouvelles recherches sont incontestablement nécessaires.

Puisque la lipase est responsable de la rancidité par l'hydrolyse qu'elle provoque, on peut penser que son action est proportionnelle à la petitesse des globules gras, c'est-à-dire à leur surface totale. Il en est bien ainsi, Dorner et WIDMER [49] ont montré que l'homogénéisation du lait et de la crème facilite fortement le rancissement. Il en serait de même avec des machines (pompes) agitant le lait trop violemment et pour la même raison.

c) Action de la chaleur sur la lipase du lait. - Il n'y a pas unanimité sur la température de destruction de la lipase du lait de vache.

Davies, dans l'étude à laquelle nous avons déjà fait allusion, estime que l'activité lipasique est arrêtée au-dessus de $62^{\circ} 5$. Pour Dorner et Widmer [49] une température à $55^{\circ}$ maintenue $20 \mathrm{mi}$ nutes suffirait à la détruire.

Leroy et $\mathrm{S}$. Palmer [48] estiment au contraire que même après 
chauffage à $75^{\circ}$ de quelques minutes, l'action de la lipase est seulement retardée en général, mais quelquefois aussi empêchée.

D'autres auteurs se contentent d'affirmer que lalipase est détruite par la pasteurisation haute, mais sans préciser de quelle température il s'agit.

d) Protection contre l'hydrolyse diastasique. - Il ressort de ce court aperçu qu'on ne peut espérer obtenir du lait eru vraiment exempt de lipase et que le meilleur moyen d'éliminer cet agent responsable de la rancidité est la pasteurisation, haute de préférence.

Notons bien qu'effectivement le rancissement du lait pasteurisé, même conservé plusieurs jours, est un fait absolument exceptionnel (1).

\section{CAS PARTICULIER D'ALTÉRATION DE LA MATIÈRE GRASSE}

Nous avons déjà parlé du goût du poisson causé par la triméthylamine. Cettesubstance, avons-nous dit, provient le plus souvent de la transformation du triméthylglyeocolle ou bétaïne, rencontré normalement dans la betterave.

Or, la triméthylamine peut très bien se former à partir de la matière grasse du lait elle-même, en suivant le processus que voiei : Il existe dans le lait une proportion non négligeable de lécithine, véritable graisse phosphorée, qui est un glycérophosphate de choline. L'oxydation de la choline conduit direotement au triméthylglycocolle (bétaïne) qui, à son tour, comme la bétaïne de la betterave, peut donner de la triméthylamine à odeur et saveur de poisson.

SuPPLEe [51] (1919) a montré que le beurre normal contenait assez de lécithine pour donner ces traces de triméthylamine responsables du goût de hareng ou de maquereau.

Ce même auteur a montré que l'acidité du milieu était un facteur nécessaire de l'apparition de la triméthylamine, mais non suffisant. Un facteur biologique doit être envisagé.

HagLUND et WaLLER [52] conseillent d'ailleurs de diminuer l'acidité de la crème pour combattre ce goût de poisson (2).

CusIck confirme les vues de SUPPLEE relatives à la transformation de lécithine en triméthylamine et note que l'apparition du goût de poisson est proportionnelle à la teneur en lécithine.

(1) Au contraire le beurre fabriqué aveo de la crème non pasteurisée, extraite du lait cru, présente très fréquemment cet inconvénient et d'une manière d'ailleurs irrégulière, en relation probable avec les saisons, e'est-à-dire avee les étapes de la lactation et la composition de la ration des vaches.

Le remède, done, serait également la pasteurisation de la crème (de préférence à assez haute température) en vue de la fabrication du beurre.

(2) Notons en passant que nous retrouvons ce souci de baratter de la crème douce pour des raisons diverses, toujours en rapport avec eertains mauvais goûts, dans les travaux de Sommer et Smith, dans ceux d'Hessenberg, dans ceux de Rocers, etc. 
Cette question n'a pas été suffisamment étudiée pour en donner ici un exposé plus complet. En particulier, le mécanisme de la transformation des lécithines en triméthylamine n'est pas précisé de façon certaine, bien que tout le monde soit d'accord pour lui attribuer une origine biologique.

La seule précaution pratique qui puisse être envisagée contre le goût de poisson, à vrai dire assez rare, est done encore la pasteurisation, assez haute pour détruire le microbe ou la diastase responsable, et la protection contre les réinfections postérieures dans l'hypothèse où cette altération aurait une origine purement microbienne.

$$
\text { *** }
$$

\section{Résumé et conclusions générales.}

Nous croyons avoir passé en revue à peu près toutes les causes possibles de saveurs et odeurs anormales dans le lait actuellement connues. Si les causes sont assez peu nombreuses, leurs effets en revanche sont extrêmement variés. Mais les remèdes généraux, communs à beaucoup de ces causes, sont eux-mêmes très peu nombreux et il est facile d'en établir la liste.

\section{ENUMERATION DES CAUSES DES SAVEURS ET ODEURS ANORMALES, \\ DE LEURS EFFETS ET DES REMEDES QU'ELLES COMPORTENT}

1. Introduction accidentelle dans le lait de substances chimiques ou pharmaceutiques, d'ingrédients divers de nettoyage ou de désinfection, ou même simplement séjour de ces substances et ingrédients à proximité du lait qui en prend la saveur et l'odeur.

Effet : odeurs et saveurs variées toujours anormales, souvent désagréables.

\section{Remèdes:}

a) Eliminer ces substances de l'étable ou de la laiterie.

b) Aérer largement les étables, la laiterie.

c) Réfriger le lait à l'air libre après pasteurisation.

2. Introduction dans la ration des plantes, fourrages ou aliments suivants :

a) légumineuses (qui donnent le goût et l'odeur de vache et de fourrage) ;

b) plantes bulbeuses à odeur forte (qui donnent un goût âcre et nauséabond) ;

c) betterave fraîche (qui donne le goût de poisson);

d) la pulpe de betterave fraîche (qui donne également le goût de poisson);

e) La pulpe de betterave fermentée sans ensilage lactique (qui 
donne le goût et l'odeur d'acide butyrique et qui est toxique pour le nourrisson);

f) les crucifères (choux, navet, colza) qui donnent le goût de la plante correspondante, souvent aussi le goût de moutarde et sont toxiques.

\section{Remèdes à ces diverses causes :}

a) Suppression pure et simple de ces aliments sauf peut-être les légumineuses dont l'ineonvénient est assez faible - constitution d'une ration à base de foin, de tourteaux autres que ceux de crucifères, de graines de céréales concassées, de son, d'avoine, de carotte, de pois, de pomme de terre, etc.

b) Usage de pulpes sèches ou soumises à l'ensilage lactique.

c) Aération du lait après pasteurisation.

3. Altérations d'originé bactériologique.

Certains ferments lactiques donnent un goût de brûlé, de malt, de caramel, d'amertume.

Certaines associations lactique-citrovorus donnent une saveur métallique. De nombreux microorganismes autres que les ferments lactiques sont responsables de saveurs anormales (amères, moisies. médicinales, phénolique, etc.).

\section{Remède général commun :}

a) Asepsie et désinfection, à la traite et à la laiterie surtout áprès pasteurisation.

b) Pasteurisation précoce à haute température.

c) Aération du lait après pasteurisation.

4. Altération d'origine physico-chimique.

Ce sont des oxydations conduisant au goût d'huilage-suiffage et qui peuvent être causées :

a) par la lumière solaire ;

b) par l'ozone ;

c) par certains sels métalliques et surtout les sels de cuivre.

\section{Remède :}

a) Eviter l'action prolongée de la lumière solaire.

b) Eviter l'emploi de l'ozone comme désinfectant.

c) Supprimer le cuivre dans l'appareillage de laiterie.

5. Altérations d'origine diastasique.

Elles sont de deux sortes :

a) Oxydation diastasique (produisant l'huilage-suiffage) qui exige la présence simultanée :

de corps gras non saturés dans la ration, 
de diastases oxydantes, de sels métalliques (cuivre surtout), et l'absence de substances réductrices.

\section{Remèdes :}

1. Supprimer dans l'alimentation des vaches les tourteaux oléagineux qui apportent des corps gras non saturés.

2. Supprimer le cuivre de l'appareillage et les ustensiles.

3. L'introduction de substances réductrices sous la forme des cultures sèches de $B$. frigidum neutrale de Kertesz peut être retenue, sous réserve de certaines études complémentaires au point de vue hygiénique et légal.

b) Hydrolyse diastasique (produisant le rancissement). Causée par la lipase et par certains microbes.

\section{Remède :}

Pasteurisation précoce et à haute température du lait.

6. Altérations diverses.

Cas particulier de l'apparition du goût de poisson à partir d'une transformation des lécithines, d'origine biologique.

\section{Remède :}

1. Pasteurisation précoce et à haute température.

2. Asepsie après pasteurisation.

Il est facile de voir que les mêmes remèdes reviennent souvent pour lutter contre des causes variées. Comme il arrive fréquemment que ces causes peuvent agir simultanément (et donner des saveurs anormales mixtes indéfinissables), la prudence conseille de se protéger à la fois contre toutes les causes actuellement connues. Nous devons done envisager d'appliquer en même temps tous les remèdes cités.

En les regroupant en fonction des divers stades de l'industrie du lait, nous obtenons le schéma suivant :

\section{A LA PRODUCTION DU LAIT}

1. Eliminer de l'étable tous les produits ou ingrédients odorants.

2. Aérer largement les étables.

3. Eliminer de l'alimentation des vaches les plantes bulbeuses, la betterave fraîche, la pulpe fraîche, les crucifères, les tourteaux.

La pulpe conservée ne sera donnée que si elle a été séchée ou ensilée avec des ferments lactiques.

Les tourteaux oléagineux ne seront donnés que si on prend soin de supprimer toute trace de cuivre dans le matériel. Mieux vaudrait les proscrire complètement. 
En revanche donner des fourrages verts (préférer les graminées aux légumineuses), du foin, des graines de céréales concassées, du son, de l'avoine, de la carotte, des pommes de terre.

4. Effectuer la traite le plus proprement et le plus aseptiquement possible.

\section{AU TRAITEMENT DU LAIT}

1. Pasteuriser le plus tôt possible après la traite et à haute température (c'est-à-dire au moins à $80^{\circ}$ ).

2. Refroidir à l'air libre pour aérer le lait et ne pas refroidir à trop basse température.

3. Eviter les réinfections après pasteurisation.

4. Eliminer toute trace de cuivre (ou alliages) même étamés dans les appareils et ustensiles divers.

5. Eviter l'action prolongée de la lumière solaire.

6. Eviter l'emploi de l'ozone comme désinfectant.

7. Eviter les machines rapides (pompes, etc.) eapables d'agiter le lait trop violemment.

8. Et éventuellement : utilisation des ferments de Kertesz.

Comme un certain nombre de ces précautions sont déjà prises dans le travail normal du lait de vente en nature, les points esseitiels qui doivent retenir l'attention de l'industriel se ramènent done à eeci :

\section{Pasteurisation haute. \\ Aération du lait.}

Suppression absolue du cuivre et de ses alliages (même étamés).

Et peut-être, sous certaines réserves, usage des ferments

de Kertesz si le cuivre ne peut être supprimé.

Telles sont les grandes prescriptions qui permettent de lutter avec succès contre la majeure partie des saveurs et odeurs anormales du lait. Elles auraient, certes, avantage à être complétées par les remèdes préconisés à la production du lait. Mais l'industriel ne peut pas toujours agir sur ses fournisseurs. Même dans ce cas regrettable et d'ailleurs général, un grand nombre d'ineonvénients parmi les plus graves seront supprimés par l'application de ces grands remèdes dans l'industrie du lait de consommation en nature.

\section{BIBLIOGRAPHIE.}

[1] Roadhouse et K a cestuer (cité par Dorner). Le Lait, 1930, p. 100.

[2] Rosengrems. Svenska Mejeritidingen, 1927, no 26.

[3] BABcoor. Bulletin no 13ะ6 du Ministre de l'Agriculture des Etats-Unis.

[4] Moнr et Воснош. Molkerei Zeituni, 1929, no 5 s̀ et 54.

[5] Posx. Pharm. Weelkblad, 1930, t. LXVII, p. 1309. 
[6] Post. Zeits. f. Unt. d. Lebensmittel, 19s1, t. LXI, p. 171.

[7] Dr Jules Renault, Conseil Sup. d'Hygiène de France.

[8] Orla-Jensen. Le Lait, 1925, p. 30.

[9] Вавсок. Un. St. Dep. of Agr. Bull, 1924, no 1279.

[10] Вавсок. Un. St. Dep. of Agr. Bull., 1923, no 1208.

[11] M. R. La Vie à la Campagne, 1923, n² 240, p. 248.

[12] EichstäDt et DibBenn. Milchwirtsch. Forsch, 19z8, t. VI, p. 564.

[13] ВАвсок. Un. St. Dep. of Agr. Techn. Bull., 1927, nº 9, p. 6.

[14] Leitch et Renwick. Bull. West of Scotland Agr. coll., 1918, p. 21-22. Glascow.

[1ii] OrLa-Jensen. Bactériologie laitière, 1921.

[16] Hammer et Cordes. Research Bull., n 68. Agr. Exp. St., Ames Iowa, 1921.

[17] SADLER. Le Lait, 1927, p. 126.

[18] BAKER et HaMmer. Bull. Rens, frigorif., mai-juin 192S, p. 468.

[19] Bull. Rens. frigorif., a oût 1928, p. 690.

[20] Ohuau. Molkerei Zeitung, 1930, t. XLIV, p. 2130.

[21] SADLER. Scientific. Agric., 1929, t. X, p. 111.

[22] SpItzen et EPPLe. Journ. of Dairy Science, 1920, t. III, p. 486.

[23] HoOD et WHITE. Congrès intern. lait., Oopenhague, 1931.

[24] Felier. Journ. of Dairy Science, sept. 1922, vol. V, p. 485.

[25] Tracy et Ruche. Journ. of Dairy Science, mai 1931, p. 250-267.

[26] WHitehead. Congrès intern. lait., Copenhague, 1931.

[27] GUthrie, Roadhouse, Hilgardia, 1931, t. V, p. 425.

[28] Wегон. Forsch. Landwirstchaft., 1932, t. VII, p. 7.

[29] Rice. Journ. of Dairy Science, 1926, t. IX, p. 459.

[30] Matrick. The Journ. of Agricult. Science, 1927, vol. XVII, p. 388.

[31] Wright. Le Lait, 1931, p. 564.

[32] SAMUtuson. Svenska Mejeritidningen, 1929, p. 275.

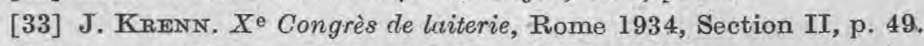

[34] Kende, MaJer. Molkerei Zeitung, 1932, nº 107.

[35] DIERnhofer. Milschw. Forschungen, 1930, vol. X, p. 319-335.

[36] Csiszar. Milschw. Forschungen, 1932, vol: XIV, p. 288-341

[37] QUam et HELLwIa. Journ. Biol. Chem., 1928, vol. LXXVIII, p. 681.

[38] W eIOH et BAUER. Fortschritte d. Ldw., 1932, p. 7-9.

[39] Grendei. Pharmac. Weekblad, 1930, p. 913 et Chem. Centrabl., 1930, p. 2453.

[40] ZBINDEN. Le Lait, 193:, p, 481.

[41] Rice. Journ. of Dairy Science, 1923, vol. IV, p. 261.

[42] QUAM. Ind. Eng. Chem., 1928, vol. XX, p. 930.

[43] Gebhard et Sommer. Ind. Eng. Chem. Ed. Anal., 1931, t. III, p. 24.

[44] PaLmer. Journ. of Dairy Science, 1926, t. IX, p. 272.

[40] K ENDE. Molk. Zeitung Hildesheim., 1931, no 102.

[46] Kende. Milch. Forschungen, 1932, n० 37.

[47] MAJER. Hildesheimer Molkereizeitung, 1931, $\mathrm{n}^{\circ} 9 \mathrm{~s}$.

[48] Jarroy et S. PALMer. The Journ. of Dairy Science, mars 1922, vol. V, no 2 d

[49] DORNER et WIDMER. Le Lait, 1931, p. 545.

[50] Proks. Zermedelsky Archiv., 1931, fasc. 1-2.

[51] Stupplee. Cornell L'niveroity Agr. Exp. Stat., ] 919.

[52] Hagiund. Le Lait, 1926, p. 206. 